UCRL-JC-122948

PREPRINT

\title{
Three Electrode Measurements on Solid Electrolytes
}

Ai Quoc Pham

Robert S.Glass
RECEIVED

MAY 021988

OSTI

This paper was prepared for submittal to the

Proceedings of the Symposium on Electrically-Based Microstructural Characterization,

Fall Meeting of The Materials Research Society

in Boston, MA, on November 27-December 1. 1995.

December 1995

This is a preprint of a paper intended for publication in a joumal or proceedings. Since chinges may be made before publication, this preprint is made available with the understanding that it will not be bited or reproduced without the permission of the wihor.

\section{MASTER}

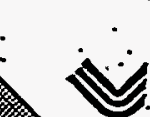




\section{DISCLAMMER}

This document was prepared as an account of work sponsored by an agency of the United States Government. Neither the United States Government nor the University of California nor any of their employees, makes any warranty, express or implied, or assumes any legal liability or responsibility for the accuracy, completeness, or usefulness of any information, apparatus, product, or process disclosed, or represents that its use would not infringe privately owned rights: Reference herein to.any specific commercial product, process, or service by trade name, trademark, manufacturer, or otherwise, does not necessarily constitute or imply its endorsement, recommendation, or favoring by the United States Government or the University of California The views and opinions of authors expressed herein do not necessarily:state or reflect those of the United States: Government or the University of.California; and shall not be used for advertising or product endorsement purposes:" 
A.Q. PHAM, R.S. GLASS

Chemistry and Materials Science Department, Lawrence Livermore National Laboratory, P. O. Box 808, Livermore, CA 94550.

\section{ABSTRACT}

$A C$ impedance spectroscopy and chronopotentiometry have been used to study solid-state ionic conductors. Results obtained using three electrodes are compared to those using a two:electrode configuration: The uncompensated resistance was shown to depend strongly on the geometric placement of the electrodes. The optimal configuration for minimized uncompensated resistance effects is similar to the Luggin capillary arrangement in the liquid phase. The effect:of non-negligible geometric capacitance on interpretation of results is discussed.

\section{INTRODUCTION}

Voltammetry using a three electrode configuration is an important and widely used technique in liquid phase electrochemistry for the characterization of electrode processes. . This technique has been extended to the solid state to study ionic conductors used in various applications, including fuel cells and sensors. In the liquid phase, there are well-established protocols which are generally used for cell design, especially. with regard to the geometric placement of the electrodes for minimization of uncompensated cell resistance effects (iR $R_{u}$ ). It is apparent from the literature that there is much less uniformity in the solid state, where virtually every conceivable configuration for the relative disposition of the working, counter and reference : electrodes has been used [1-3]. In some cases, the uncompensated resistance effects were simply ignored [1]. In other cases, it was determined using the current initerruption technique or two or: thiree-probe Electrochemical Impedarice Spectroscopy (EIS) [2,3]: However, the validity. of the experimental methods used for $\mathrm{iR}_{\mathbf{u}}$ correction has not been rigorously examined. In addition, unlike the case for liquid electrolytes, solid materials often have a non-negligible geometric capacitarice. This capacitive component may. generate problems that do not exist in the liquid phase.

In this paper, we have used EIS and chronopotentiometry to examine the influence of the geometric placement of the electrodes on the uncompensated resistance. Comparison of methods to determine $R_{u}$ has been made. The effect of non-negligible geometric capacitance has also been examined. Optimal cell designs for obtaining data on solid electrolytes is considered.

\section{Principles of three-electrode measurements:}

$\because:$ In three electrode voltammetric measurements in solution, the potential of the working electrode is controlled versus the reference electrode, which is assumed to have constant potential. Current is passed between the counter and working electrodes. Not all the potential measured between the working and reference electrode is due to potential drops at the electrode/solution interfaces. There is always some finite potential drop resulting from uncompensated solution resistance $\left(\mathrm{iR}_{\mathrm{W}}\right)$.

For solid electrolytes using pellets or films, the reference electrode is placed either on the same side as the working or counter electrode. The reference electrode is often made of the same material as the working electrode, and, therefore, it does not have a fixed potential. The potential of the reference electrode is determined by the themodyriamic equilibria established between the solid electrolyte, the electrode and the gaseous environment for a given temperature. In the absence of current flow, the overpotential measured between the working and reference electrode can be written:

$$
\eta=E_{W}-E_{\text {xef }}
$$

In the presence of current flow, there is the additional factor of uncompensated resistance. which must be included. In this case, the overpotential is given by: 


$$
\eta=E_{W}-E_{R e r}-i R_{u}
$$

Because the reference electrode is placed in the same plane as the working or counter electrode, erroneous assumptions have sometimes been made about the magnitude of the uncompensated resistance. When it is has been placed in the same plane as the. working electrode, $R_{u}$ has often been assumed to be negligible and the measured potential difference given by ( 1 ). When it is placed on the opposite side, $R_{\mathbf{u}}$ is assumed to be equal to the total electrolyte resistance between the working and counter electrodes. As will be shown, this can lead to significant errors.

\section{EXPERIMENTAL}

Yttria-stabilized zirçonia (YSZ, $8 \mathrm{~mol} \%$ ) from Tosho was used as the solid electrolyte. Sintered pellets having a diameter of $8 \mathrm{~mm}$ and thickness of $1.5 \mathrm{~mm}$ were used for all measurements. Silver paint was applied on both sides of the ceramic disks to make electrodes.

In order to examine the effect of electrode placement, three different cell configurations were used, as shown in Figure 1. On pellet $I$, the reference electrode (A) was placed as far as possible $(3 \mathrm{~mm}$ ) from electrode $B$. On pellet $\Pi$, the reference electrode was placed as close as possible to electrode $B(0.5 \mathrm{~mm})$. For both pellets $I$ and $I$, the reference electrode had a width of $1 \mathrm{~mm}$. The third pellet (III), had an electrode arrangement resembling that of the Luggin capillary configuration in liquid phase electrochemistry. The reference electrode was in form of a small spot, $1 \mathrm{~mm}$ in diameter, equidistant $(0.5 \mathrm{~mm})$ to the surrounding electrode $B$.

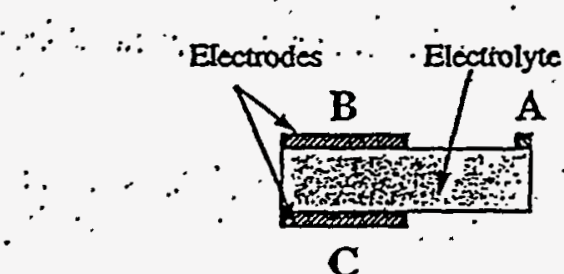

pellet I.

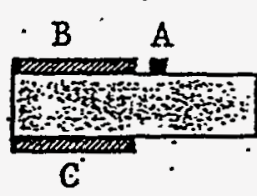

pellet II

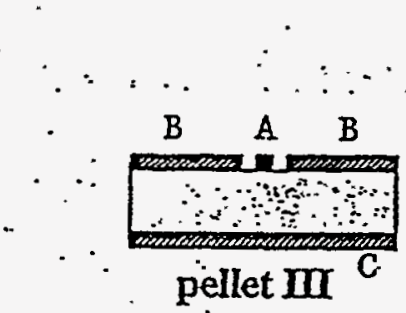

pellet III

Figure 1: Electrode placement on YSZ ceramic disks.

EIS measurements were performed using both two-probe and three-probe configurations. For these measurements, a Solartron Frequency Response Analyzer Model 1255 was coupled with an EG\&G model 273 potentiostat. The latter instrument was also used for chronopotentiometric measurements. In two-probe measurements, the reference and counter electrode connections are short-circuited and connected to electrode $\mathrm{C} ; \mathrm{B}$ is then the working electrode. In three-probe measurements, the reference electrode is connected to $\mathrm{A}$, and $\mathrm{B}$ and $\mathrm{C}$ are the working and coünter electrodes, or vice versa:

\section{RESULTS AND DISCUSSION}

Figure 2 shows the impedance spectra corresponding to various electrode configurations for pellet $I$ in air at $700^{\circ} \mathrm{C}$. Plot "a" corresponds to the classic two-probe measurement where only electrodes $\mathrm{B}$ arid $\mathrm{C}$ are used: At such a high temperature; the bulk electrolyte capacitance is negligible and the corresponding equivalent circuit element for the electrolyte is reduced to a simple resistor having a resistance of $32 \Omega$ (the low frequency intercept). The response of both electrodes $\mathrm{B}$ and $\mathrm{C}$ contributes to the observed spectrum; the total electrode resistance, measured between the low and high frequency intercepts with the real axis, is $16 \Omega$ :

Plot " $b$ " in Figure 2 represents the situation when $B$ is used as the working electrode, $C$ is the counter electrode, and $\mathrm{A}$ is used as the reference. In this case, a spectrum similar in shape to plot "a" was.obtained. However, a mụch smaller electrode resistance is observed $(7 \Omega)$ because only the contribution from electrode $B$ is measured. The high frequency part of the spectrum 
intercepts the real axis at $12 \Omega$. This value corresponds to the uncompensated resistance between the working and reference electrodes for this cell configuration. The non-zero value indicates that although the reference electrode is in the same plane as the working electrode, the surface -just beneath the two electrodes are not on the same equipotential lines.

Reversing the working and counter electrode connections above, that is; using $C$ as the . working electrode, results in a higher measured value of the uncompensated resistance, $20 \Omega$ (plot " $c$ "). This value is, however, smaller than the bulk electrolyte resistance measured between $\mathrm{B}$ and $\mathrm{C}$ in plot " $\mathrm{a}$." Therefore, the uncompensated resistance when the refererice and working electrodes are on opposite sides of the pellet does not correspond to the resistance determined by two-probe measurements.

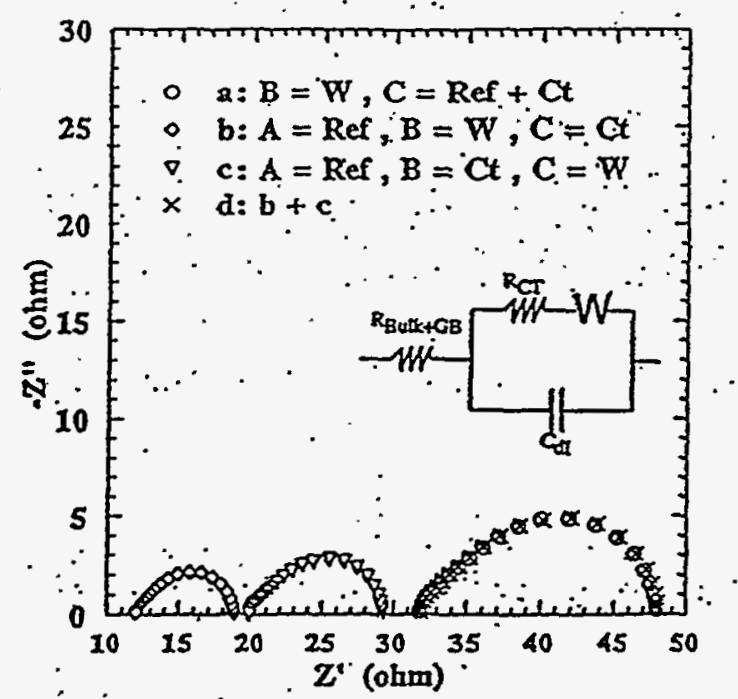

Figure 2 : Impedance data in air ät $700^{\circ} \mathrm{C}$ for pellet $\mathrm{I}$ :

Interestingly, the sum of the two uncompensated resistances in plot " $b$ " and " $c$ " corresponds to the total electrolyte resistance measured between $B$ and $C(32 \Omega)$ determined in plot " $\mathrm{a}$ " This feature clearly indicates that the reference electrode $A$ is at a potential intermediate to those of B and C. The sum of the impedance spectra " $b$ " and " $c$ " (plot " $d$ ") also matched. closely" that of the two-probe impedance plot " $a$ " ${ }_{3}$.confirming that the thiree-probe measurements truly measure the processes occurring at only one electrode. The addition of the impedance plots was done by adding the $Z$ ' and $Z$ ' values of the two spectra at each frequency.

The interpretation of the origin of uncompensated resistance is well-understood in liquid. phase electrochemistry. Using computer simulation, Landau, et al., have studied. the equipotential line distribution for various cell configurations (4). An analogy can be made in the: solid.state. In a qualitative fashion,. Figure 3 shows the equipotential line distribution inside the electrolyte for the specific.case of pellet I.(quantitative data will be published elsewhere (5)). Between the working and counter electrodes, where the current is flowing, the equipotential lines: are parallel to each other and parallel to the electrode surfaces. Outside of this region, the equipotential lines bend towards the edges of the electrodes. The density of the equipotential lines is very high .close to the.edges and decreases rapidly as one moves away from the electrodes. Therefore, a reference electrode placed somewhere on the electrolyte surface in the same plane as the working or counter electrodes will never be on the same equipotential as these electrodes... The intermediate value (greater than zero. but smaller than the total bulk resistance): of the measured uncompensated resistance appears as an immediate consequence if is to be emphasized that this resistance is not the -resistance between the working and the reference electrodes as is often enorieously assumed. It is part of the total electrolyte resistance between the working and the counter electrodes (see Figure 3). For the parallel plate electrode configuration this point constitutes one of the major differences between liquid and solid phase electrochemistry. 


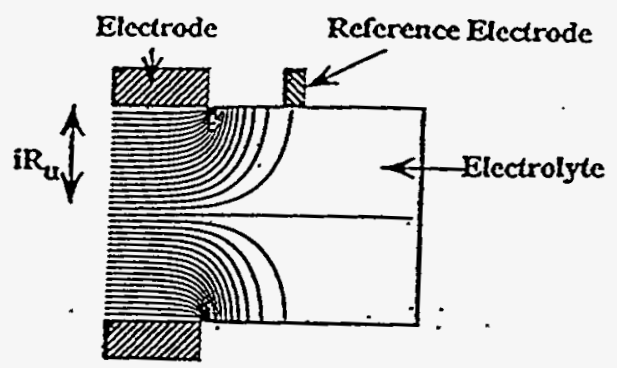

Figure 3: Equipotential line distribution in the solid electrolyte.

Influence of the geometric capacitance:

In the liquid phase, the solution ustally has high conductivity and the bulk electrolyte equivalent circuit element is a simple resistor. In the solid state, an analogous situation usually occurs only at high temperatures and only for some electrolytes where ionic conductivity is appreciable. At lower temperatures, the equivalent circuit for the bulk electrolyte is a resistor in parallel with the geometric capacitance.

Figure 4(a) shows the impedance spectra recorded at $400^{\circ} \mathrm{C}$. At this temperature, in the two-probe mode, the semicircle corresponding to the grain boundary relaxation is clearly visible. While it is clearly present in Figure 4(a), the semicircle corresponding to the bulk electrolyte is not.completely resolved because of the high frequency limitation of the instrumentation used (100 kHz). In contrast, in three-pröbe ac measurements (Figure.4(b)); half of the bulk semicircle is visible within the same frequency range. This result requires some interpretation (see below).

In contrast to the results obtained at higher temperature (Figure 2), the sum of the two three-probe mode spectra in Figure 4(b) did not yield the same piot as the two-probe one (see .Figure 4(a), plot $(V)$. The problem may arise from the fact that in the three-probe mode, the voltage, which is applied between the reference and working electrodes, is not associated with the total capacitance as is the current. It is actually only associated with the slice of the electrolyte that determines the uncompensated resistanice. Caution is therefore required when interpreting three-probe EIS measurements if the sample has a non-negligible capacitance in parallel with the resistance. The presence of the semicircles attributed to the bulk and grain boundary conduction indicates once again that the three-probe mode not only measures the working electrode process but part of the electrolyte conduction as well.

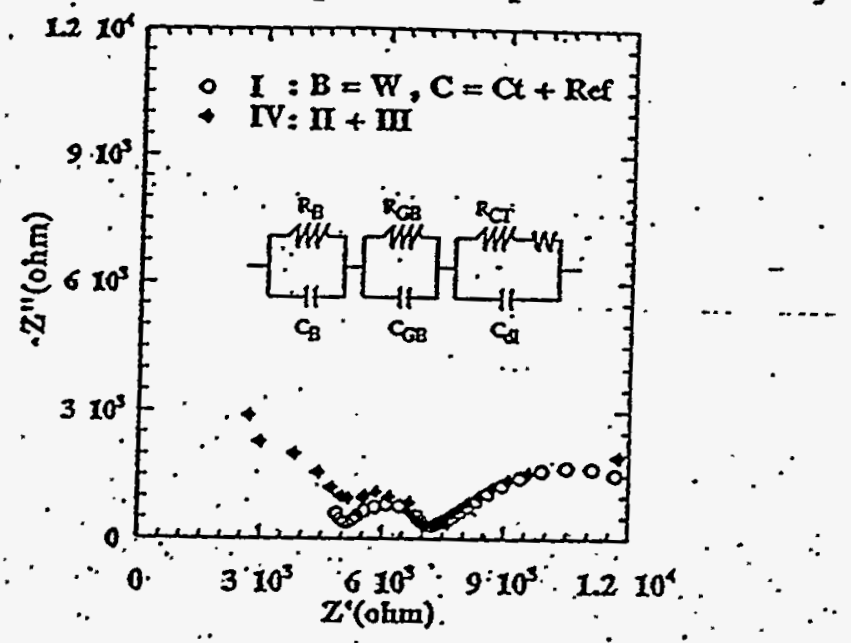

a

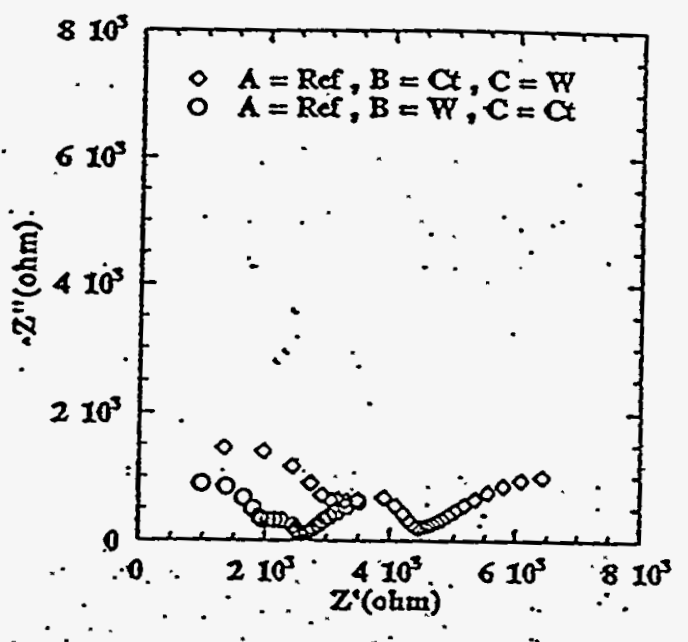

b. 


\section{Current sicn measurements for the determination of the uncompensated resistance:}

The current interruption method utilizes the difference between relaxation time constants to separate slow and fast processes. When the electrolyte is a pure resistor, the time constant is very small, and when the current is interrupted, the potential will drop instantaneously. The time constant associated with the electrode interface is usually much more important because the capacitance is higher, and the corresponding potential will decrease slowly with time.

Equivalent to the current interruption method is the current step technique. In this technique, the current is suddenly increased from zero to a finite value. The potential will jump rapidly for fast processes and then increase more slowly for slower ones. Because. of instrumental limitations, we chose to acquire data using the current step technique.

Figure 5 shows the results of current step measurements for different electrode configurations. The resistance obtained using the two-probe measurement was $36 \Omega$, almost equal to the bulk electrolyte resistance determined previously by the two-probe EIS measurement $(32 \Omega)$. In the three-probe configuration, the measured uncompensated resistance was 14 and 23 $\Omega$ when the reference electrode $A$ is on the same side $(B=$ working) and on the opposite side (C $=$ working) of the pellet, respectively. Once again, these values are close to the ones measured -by. EIS (Table $\mathrm{D}$, confirming the equivalence of the two techniques.

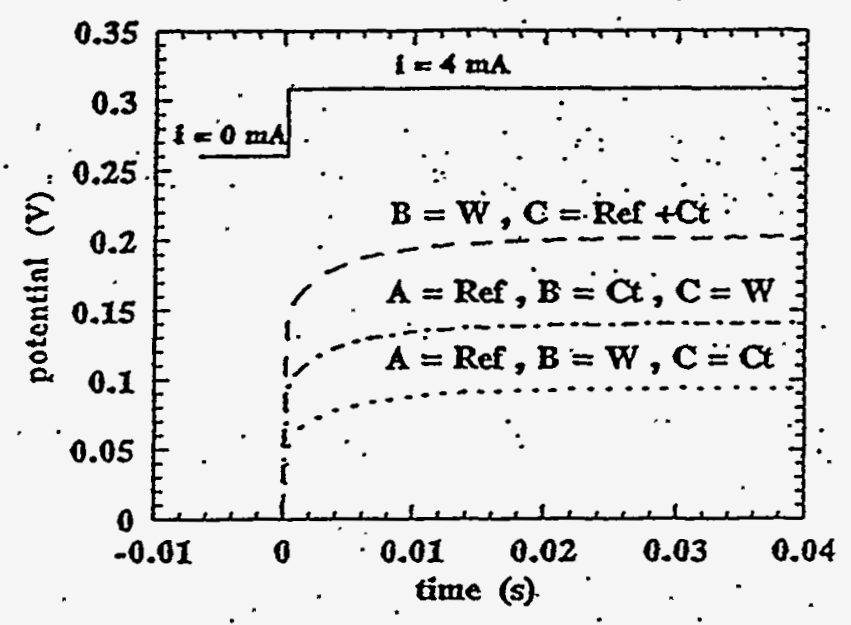

Figure 5: Chronopotentiometric data recorded at $700^{\circ} \mathrm{C}$. Step time resolution $=0.5 \mathrm{~ms}$.

Table I : Uncompensated resistance (in $\Omega$ ) as determined by three-probe EIS $(A C)$ and by the current step (CS) technique.

\begin{tabular}{|c|c|c|c|c|}
\hline$\therefore$ & \multicolumn{2}{|c|}{$700^{\circ} \mathrm{C}$} & \multicolumn{2}{|c|}{$400^{\circ} \mathrm{C}$} \\
\hline Electrode configuration & RCS. & $\overline{\mathrm{R}_{\mathrm{AC}}}$ & $\therefore \mathrm{R} \dot{\mathrm{CS}}$ & $\overline{R_{A C}}$ \\
\hline $\begin{array}{l}B=W, C=C t+\operatorname{Ref} \\
A=\operatorname{Ref}, B=W, C=C t \\
A=\operatorname{Ref}, B=C t, C=W\end{array}$ & $\begin{array}{l}36^{\circ} \\
14 \\
23\end{array}$ & $\begin{array}{l}32 \\
12 \\
20\end{array}$ & $\begin{array}{r}7050 \\
\therefore \quad 2530 \\
\quad 4530\end{array}$ & $\begin{array}{l}7000 \\
2500 \\
4500\end{array}$ \\
\hline
\end{tabular}

: Experiments at $400^{\circ} \mathrm{C}$ indicated that the technique is still applicable despite the presence of the capacitive component. Experiments at these low temperatiures are possible because the grain boundary capacitance is usually three orders of magnitude smaller than that of the electrode interface; and the bulk electrolyte capacitance is about six orders of magnitude smaller. The associated time: constants are then-respectively 3 and 6 orders of magnitude smaller than the electrode relaxation time constant, and are therefore negligible when the measurement time resolution is not smaller than $0.5 \mathrm{~ms}$. This result suggests an important precaution when one applies the current interruption or current step technique. That is, the time resolution of the technique should be set appropriately according to the processes which need to be separated. 
The uncompensated resistance determined for various electrode configurations is compared in Table II. Because the electrode area is not the same for all the pellets, in order to make the comparison, the values reported are normalized to the total electrolyte resistance between $\mathrm{B}$ and $\mathrm{C}$ as determined using two-probe measurements. Noting the similarity of results for configurations $I$ and $\Pi$, it is concluded that the uncompensated resistance is weakly dependent on the distance between the reference electrode $A$ and electrode $B$. This weak dependence can be explained by considering the equipotential lines. As mentioned above, the density of the equipotential lines is very high close to the edges of the electrodes and rapidly decreases as one moves away from the electrodes. Therefore, the potential drops quickly as one moves away from the working electrode and does not change significantly after only a very short distance. Theoretically, at an infinite distance, the potential would drop by one half of the potential difference between $B$ and $C$. The width of the reference electrode. therefore does not . perturb the equipotential line distribution.

Table II : Normalized uncompensated resistance for different electrode configurations

\begin{tabular}{|l|c|c|c|}
\hline Electrode configuration & Pellet I & Pellet II & Pellet III \\
\hline $\mathrm{B}=\mathrm{W}, \mathrm{C}=\mathrm{Ct}+\mathrm{Ref}$ & 1 & 1 & 1 \\
$\mathrm{~A}=\mathrm{Ref}, \mathrm{B}=\mathrm{W}, \mathrm{C}=\mathrm{Ct}$ & $0.38 \quad:$ & 0.36 & 0.09 \\
$\mathrm{~A}=\operatorname{Ref}, \mathrm{B}=\mathrm{Ct}, \mathrm{C}=\mathrm{W}$ & 0.62 & $0.6 \dot{4}$ & 0.91 \\
\hline
\end{tabular}

In analogy to solution phase electrochemistry, it is clear that the reference electrode should be placed as close as possible to the working electrode in order to minimize the uncompensated resistance. Using conventional electrode painting techniques, it was not possible to place A closer than $0.5 \mathrm{~mm}$ to electrode $B$ without having a short-circuit. Under these constraints, we found that the configuration represented by pellet III offered the smallest uncompensated resistance (see Table II). In this configuration, because of the surrounding effect of the working electrode, the potential surface passing through the reference electrode is very close to that of the working electrode (4). The configuration of pellet III is similar to that of the Luggin capillary electrode configuration in solution electrochemistry.

\section{CONCLUSIONS}

'The ancompensated resistance in solid electrolytes has been shown to depend strongly on the geometric placement of the electrodes. The optimial configuration for minimization of the uncompensated resistance consists of a small spot reference electrode placed as close as possible to and surrounded by the working electrode. This configuration is similar to the Luggin capillary electrode configuration in liquid phase. The influence of the uncompensated resistance on voltammetry and electrolyte/electrode characterization will be published in greater detail elsewhere (5).

Three-electrode EIS has been shown to be a equivalent to the current interruption method for determination of the uncompensated resistance. For electrolytes having low conductivity, because of the presence of the capacitive component, caution in interpretation of data is suggested.

\section{REFERENCES}

1. T. Chao, K.J. Walsh and P.S. Fedkiw, Solid State Ionics 47, 277 (1991).

2. I.C. Vinke, B.A. Boukamp, K.J. de Vries and A.J. Burggraaf, Solid State Ionics 51, 249 (1992).

3. E.J.L. Scouler and M. Kleitz, J. Electrochem. Soc. 134, 1045, 1987.

4. U. Landau, N.L. Weinburg, E. Gileadi, J. Electrochem. Soc. 135, 396, 1988.

5. A.Q. Pham, R.S. Glass and F. Garzon (to be published).

*Work performed under the auspices of the U.S. Department of Energy by the Lawrence Livermore National Laboratory under contract number W-7405-ENG-48. 


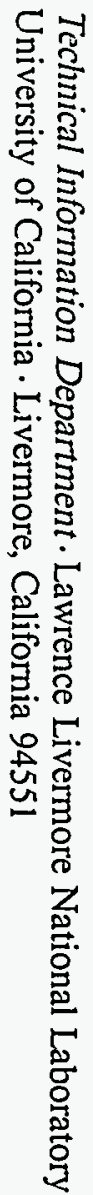

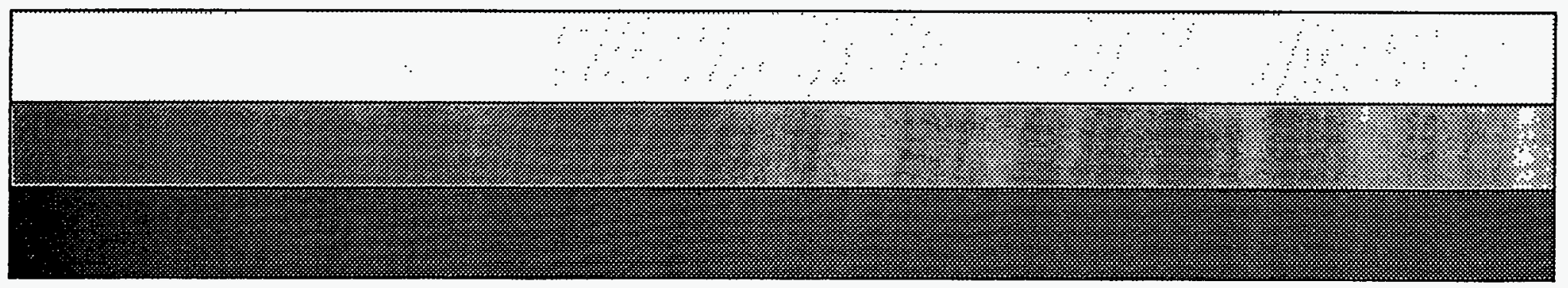

